\title{
Clinical Impact of Hemorheology on Subclinical Myocardial Injury in Patients with Hypertension
}

\author{
Takashi Hitsumoto ${ }^{\mathrm{a}, \mathrm{b}}$
}

\begin{abstract}
Background: The blood concentration of high-sensitivity cardiac troponin $\mathrm{T}$ (hs-cTnT) is a useful biomarker for myocardial injury or the pathogenesis of hypertension. Little is known about the relationship between hemorheology and myocardial injury in patients with hypertension. This cross-sectional study aimed to clarify the clinical impact of hemorheology on subclinical myocardial injury assessed with a microchannel array flow analyzer (MC-FAN) and its impact on hs-cTnT in patients with hypertension.
\end{abstract}

Methods: A total of 447 outpatients (men: 181; women: 266; mean age: $65 \pm 13$ years), with no history of cardiovascular disease, including admission for heart failure, who were undergoing treatment for hypertension, were enrolled. Whole blood passage time (WBPT) as a marker of hemorheology was measured with a MC-FAN, and the relationship between hs-cTnT levels and various clinical parameters, including WBPT, was examined.

Results: hs-cTnT levels were detected in 400 patients $(89.5 \%)$. WBPT was significantly higher in patients with detectable hs-cTnT levels than in those with undetectable hs-cTnT levels $(60.5 \pm 16.8$ $\mathrm{s}$ versus $50.2 \pm 14.2 \mathrm{~s}, \mathrm{P}<0.001)$. In patients with detectable hscTnT levels, there was a significant positive correlation between WBPT and hs-cTnT level $(\mathrm{r}=0.33 ; \mathrm{P}<0.001)$. Multiple regression analysis revealed that WBPT was an independent variable when hs-cTnT was a subordinate factor $(\beta=0.15 ; \mathrm{P}<0.01)$. Receiveroperating characteristic curve analysis indicated that a cutoff value for WBPT of $55.6 \mathrm{~s}$ yielded the largest area under the curve $(0.744$; $\mathrm{P}<0.001$ ) for discriminating high hs-cTnT levels as $\geq 0.014 \mathrm{ng} /$ $\mathrm{mL}$.

Conclusion: The results indicate that WBPT is independently associated with hs-cTnT in hypertensive patients with no history of cardiovascular events, suggesting that impairment of hemorheology in small cardiac vessels causes subclinical myocardial injury. In addition, the study suggests that progression of myocardial injury can be prevented by maintaining WBPT at approximately $\leq 55 \mathrm{~s}$.

Manuscript submitted October 5, 2018, accepted October 16, 2018

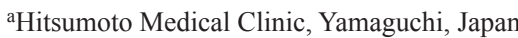

bCorresponding Author: Takashi Hitsumoto, Hitsumoto Medical Clinic, 2-7-7,

Takezakicyou, Shimonoseki-City, Yamaguchi 750-0025, Japan.

Email: thitsu@jcom.home.ne.jp

doi: https://doi.org/10.14740/jocmr3652
Keywords: Hemorheology; Microchannel array flow analyzer; Highsensitivity troponin T; Advanced glycation end products; Cardio-ankle vascular index; Oxidative stress; Hypertension

\section{Introduction}

Recent clinical studies have demonstrated that the blood concentration of cardiac troponin $\mathrm{T}$ (high-sensitivity cardiac troponin $\mathrm{T}$ (hs-cTnT)) can be measured by a highly sensitive assay. The blood concentration of hs-cTnT is a useful biomarker to evaluate myocardial injury or to predict cardiovascular events at the clinical stage [1]. In addition, several studies have reported the clinical significance of hs-cTnT in patients with hypertension [2-5].

Impairment of hemorheology is an important risk factor for cardiovascular disease as well as atherosclerosis $[6,7]$. In recent years, the microchannel array flow analyzer (MCFAN), which is a commercial device that assesses hemorheology using microscopic images, has been introduced in clinical settings [8]. MC-FAN has a simple methodology and is superior to other methods in the accuracy of channel dimensions and high reproducibility. Clinical studies have demonstrated relationships between increased whole blood passage time (WBPT), measured with MC-FAN, and cardiovascular risk factors or cardiovascular events [9-12].

Little is known about the relationships between hemorheology and myocardial injury in patients with hypertension. This study aimed to elucidate the association of WBPT with hs-cTnT in patients with hypertension and without apparent cardiovascular disease, including heart failure.

\section{Patients and Methods}

\section{Study population}

This study was conducted at the Hitsumoto Medical Clinic in Shimonoseki City, Japan, between March 2016 and February 2018. The study population comprised 447 outpatients (males: $181(40.5 \%)$ and females: 266 (59.5\%); mean age: $65 \pm 13$ years) undergoing treatment for hypertension. No patient had a history of cardiovascular events, such as coronary artery, cerebrovascular, or perivascular disease or admission for heart 


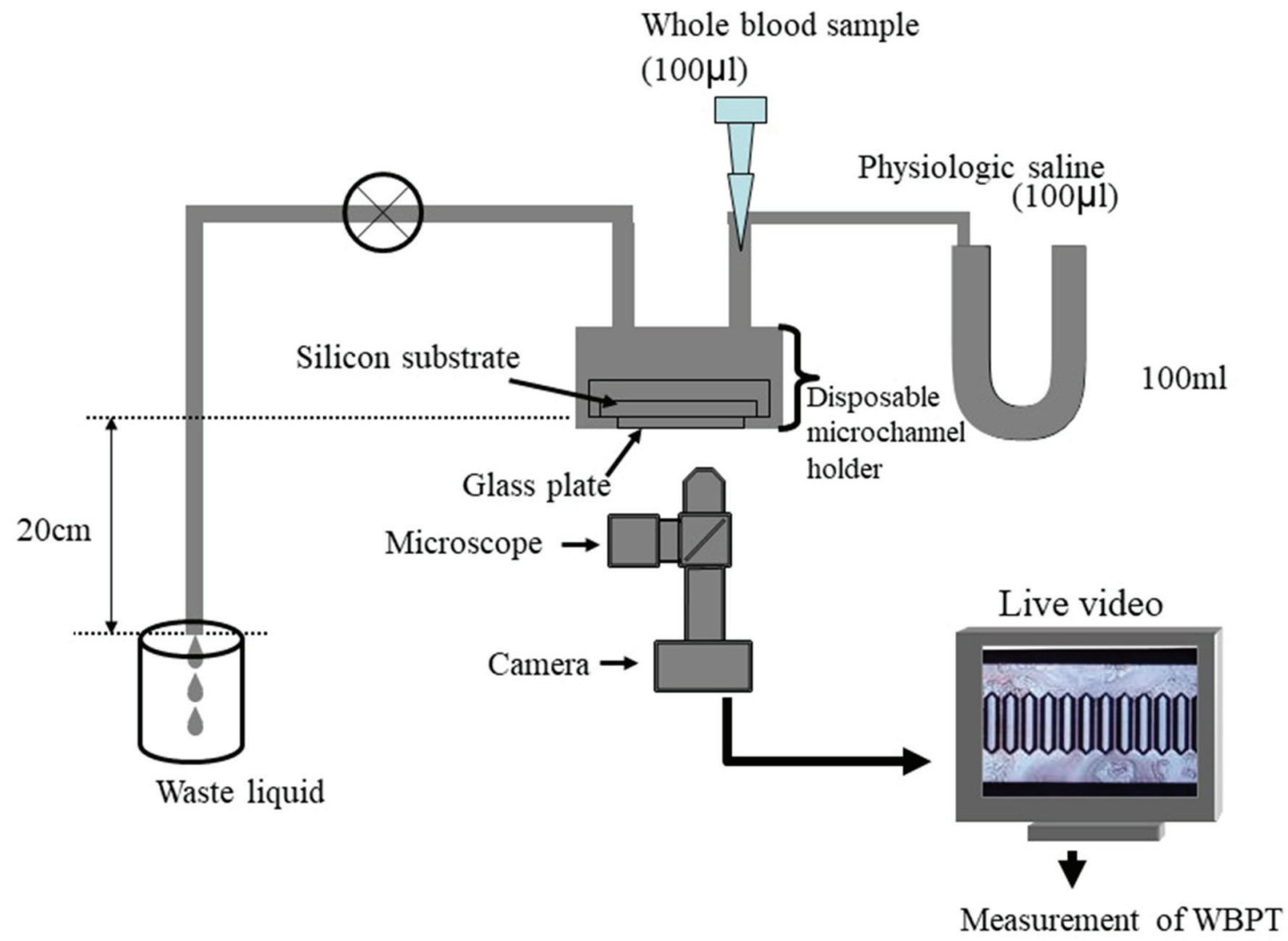

Figure 1. System of microchannel array flow analyzer. The microchannel passage time of $100 \mu \mathrm{L}$ of physiological saline was measured as a control, and then that of venous whole blood obtained from the subjects with $5 \%$ heparinization was determined. The WBPT of the subjects was expressed after correction for the passage time of physiological saline. Inter- and intra-assay coefficients of variation for WBPT were $8 \%$ and $5 \%$, respectively. WBPT: whole blood passage time.

failure. All patients provided informed consent, and the study protocol was approved by the Local Ethics Committee of the Hitsumoto Medical Clinic (approval number: 2016-02).

\section{Evaluation of hemorheology by MC-FAN}

The evaluation of hemorheology was performed by measuring WBPT with an MC-FAN HR300 rheometer (MC Healthcare, Tokyo, Japan), as previously reported (Fig. 1) [8, 10]. Briefly, the microchannel passage time for $100 \mu \mathrm{L}$ of physiological saline as a control was measured, followed by the microchannel passage time for $100 \mu \mathrm{L}$ of a heparinized whole blood sample obtained from a patient. The WBPT for patients was corrected for the passage time of physiological saline. The microchannel formation was $7-\mu \mathrm{m}$ wide, $30-\mu \mathrm{m}$ long and $4.5-\mu \mathrm{m}$ deep. WBPT was measured within $60 \mathrm{~min}$ of blood sampling. The inter- and intra-assay coefficients of variation for WBPT were $8 \%$ and $5 \%$, respectively.

\section{Estimation of clinical parameters}

Various clinical parameters, classic coronary risk factors, exercise habits, glucose-related parameters, blood cell count, kidney function, left ventricular hypertrophy estimated by electrocardiography, brain natriuretic peptide (BNP), oxidative stress, arterial function and hs-cTnT levels were evaluated. The degree of obesity was evaluated by the body mass index, calculated as the weight in kilograms divided by the height in square meters. Exercise habits were considered positive for those who performed aerobic exercise for more than 30 min three times a week. Current smoking was defined as smoking at least one cigarette per day during the previous 28 days. Treatment with antihypertensive drugs was stopped 24 $\mathrm{h}$ or more before measurement, and right brachial blood pressure was measured twice with a mercurial sphygmomanometer with the patient in a sitting position. The average of two readings was used to determine systolic and diastolic blood pressure. Dyslipidemia was defined as low-density lipoprotein cholesterol level $\geq 140 \mathrm{mg} / \mathrm{dL}$, high-density lipoprotein cholesterol level $\leq 40 \mathrm{mg} / \mathrm{dL}$, triglyceride level $\geq 150 \mathrm{mg} / \mathrm{dL}$ or the use of antidyslipidemic medication. Skin autofluorescence (AF) as a marker of advanced glycation end products (AGEs) in the tissues was measured with a commercial instrument (AGE ReaderTM, DiagnOptics, Groningen, The Netherlands), as described previously [13]. Briefly, AF was defined as the average light intensity per nanometer in the range between 300 and $420 \mathrm{~nm}$. AF levels were expressed in arbitrary units. All measurements were taken at the volar side of the lower 
arm, approximately $10-15 \mathrm{~cm}$ below the elbow fold, with the patient in a seated position. The severity of left ventricular hypertrophy was evaluated using Cornell ( $\mathrm{R}$ wave in $\mathrm{aVL}+$ $\mathrm{S}$ wave in V3) electrocardiographic voltage calculations [14]. Arterial function was evaluated by the cardio-ankle vascular index (CAVI) using a VaSera CAVI instrument (Fukuda Denshi, Tokyo, Japan) according to previously described methods [15]. Briefly, brachial and ankle pulse waves were determined with inflatable cuffs by maintaining the pressure between 30 and $50 \mathrm{~mm} \mathrm{Hg}$ to ensure a minimal effect on systemic hemodynamics from the pressure of the cuffs. Blood pressure and pulse pressure were simultaneously measured with the patient in the supine position. CAVI was measured after the patient rested for $10 \mathrm{~min}$ in a quiet room. CAVI was calculated by the formula $\mathrm{CAVI}=\mathrm{a}\left((2 \rho / \Delta \mathrm{P}) \times \ln (\mathrm{Ps} / \mathrm{Pd}) \mathrm{PWV}^{2}\right)+\mathrm{b}$, where a and $\mathrm{b}$ are constants, $\rho$ is blood density, $\Delta \mathrm{P}$ is Ps - Pd, Ps is systolic blood pressure, $\mathrm{Pd}$ is diastolic blood pressure and $\mathrm{PWV}$ is pulse wave velocity.

\section{Evaluation of blood parameters}

Blood samples were collected from the antecubital vein in the morning after $12 \mathrm{~h}$ of fasting. Total cholesterol and triglyceride concentrations were measured by standard enzymatic methods. Serum high-density lipoprotein cholesterol concentrations were measured by selective inhibition, and serum low-density lipoprotein cholesterol concentrations were measured by the Friedewald equation [16]. Patients with a serum triglyceride concentration $\geq 400 \mathrm{mg} / \mathrm{dL}$ were excluded, considering the accuracy of this method. Glucose and insulin concentrations were measured by the glucose oxidase method and enzyme immunoassay, respectively. To measure insulin resistance, homeostatic model assessment of insulin resistance (HOMA-IR) was calculated as follows [17]: HOMA-IR = fasting glucose concentration $(\mathrm{mg} / \mathrm{dL}) \times$ fasting insulin concentration $(\mu \mathrm{g} /$ $\mathrm{mL}) / 405$. Estimated glomerular filtration rate (eGFR) was calculated by the adjusted Modification of Diet in Renal Disease Study equation, which was proposed by the working group of the Japanese Chronic Kidney Disease Initiative [18]. The blood concentration of BNP was measured with a commercial kit (SHIONOSPOT Reader; Shionogi \& Co., Osaka, Japan). The derivatives of reactive oxygen metabolites (d-ROMs) test as a marker of oxidative stress in vivo was conducted with a commercial kit (Diacron, Grosseto, Italy) [19]. hs-cTnT levels were also measured with a commercial kit (Roche Diagnostics, Basel, Switzerland) [20]. In the hs-cTnT assay, the lower limit of detection was $0.003 \mathrm{ng} / \mathrm{mL}$.

\section{Statistical analysis}

The data were analyzed by Stat View-J 5.0 (HULINKS, Tokyo, Japan) and MedCalc for Windows version 14.8.1 (MedCalc Software, Ostend, Belgium). The data are expressed as means $\pm \mathrm{SD}$. Between-group comparisons were performed by Student's $t$-test or the Mann-Whitney $U$ test, and the correlation coefficient was estimated by Spearman rank-order correla- tion analysis. Multivariate analysis was conducted by multiple regression analysis. Receiver-operating characteristic (ROC) curves were constructed, and the Youden index was used to determine the optimal cutoff for WBPT for determining high hs-cTnT levels. A P value $<0.05$ was considered to indicate statistical significance.

\section{Results}

Table 1 presents the patients' characteristics. hs-cTnT levels were detected in 400 patients $(89.5 \%)$. Age, systolic blood pressure, presence of diabetes mellitus, fasting blood glucose level, HOMA-IR, skin AF, Cornell voltage, BNP, d-ROMs test, CAVI and frequency of antidiabetic medication were significantly higher and eGFR was significantly lower in patients with detectable hs-cTnT levels. WBPT was significantly higher in patients with detectable hs-cTnT levels. Correlations among hs-cTnT level, WBPT and various clinical parameters in patients with detectable hs-cTnT levels are presented in Table 2. Gender, age, presence of diabetes mellitus, fasting blood glucose level, skin AF, eGFR, Cornell voltage, BNP, d-ROMs test and CAVI were significantly correlated with hscTnT levels. Body mass index, exercise habits, current smoking status, presence of diabetes mellitus, skin AF, white blood cell count, hematocrit, eGFR, d-ROMs test and CAVI were significantly correlated with WBPT. The correlations between hs-cTnT level and WBPT are shown in Figure 2. Significantly positive correlations were observed between the two parameters. Table 3 shows the results of multiple regression analysis for hs-cTnT levels or WBPT as a subordinate factor. Explanatory factors were selected either by checking multicollinearity among variables or by the stepwise method. Skin AF, CAVI, WBPT, age, d-ROMs test, eGFR and BNP level were selected as independent variables when hs-cTnT level was used as a subordinate factor; CAVI, hs-cTnT level, d-ROMs test, skin $\mathrm{AF}$, hematocrit, exercise habits and current smoking status were selected as independent variables when WBPT was used as a subordinate factor. Figure 3 shows the ROC curve analysis for detection of high hs-cTnT levels as $\geq 0.014 \mathrm{ng} / \mathrm{mL}$ based on WBPT. A WBPT cutoff of $55.6 \mathrm{~s}$ yielded the largest area under the curve, 0.744 (95\% confidence interval: 0.699 $-0.784 ; \mathrm{P}<0.001$ ), indicating a sensitivity of $85.7 \%$ and a specificity of $57.0 \%$ for discriminating hs-cTnT levels as $\geq$ $0.014 \mathrm{ng} / \mathrm{mL}$.

\section{Discussion}

Yagi et al reported that the mean WBPT in healthy controls with no history of traditional cardiovascular risk factors, such as hypertension, hypercholesterolemia and diabetes mellitus,

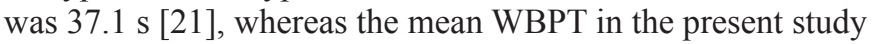
was $59.4 \mathrm{~s}$. Thus, these results indicate that the impairment of hemorheology in hypertensive patients was progressive compared with that in healthy controls. Furthermore, independent associations between WBPT and hs-cTnT levels in this study suggest that the impairment of hemorheology affects the pro- 
Table 1. Patient Characteristics

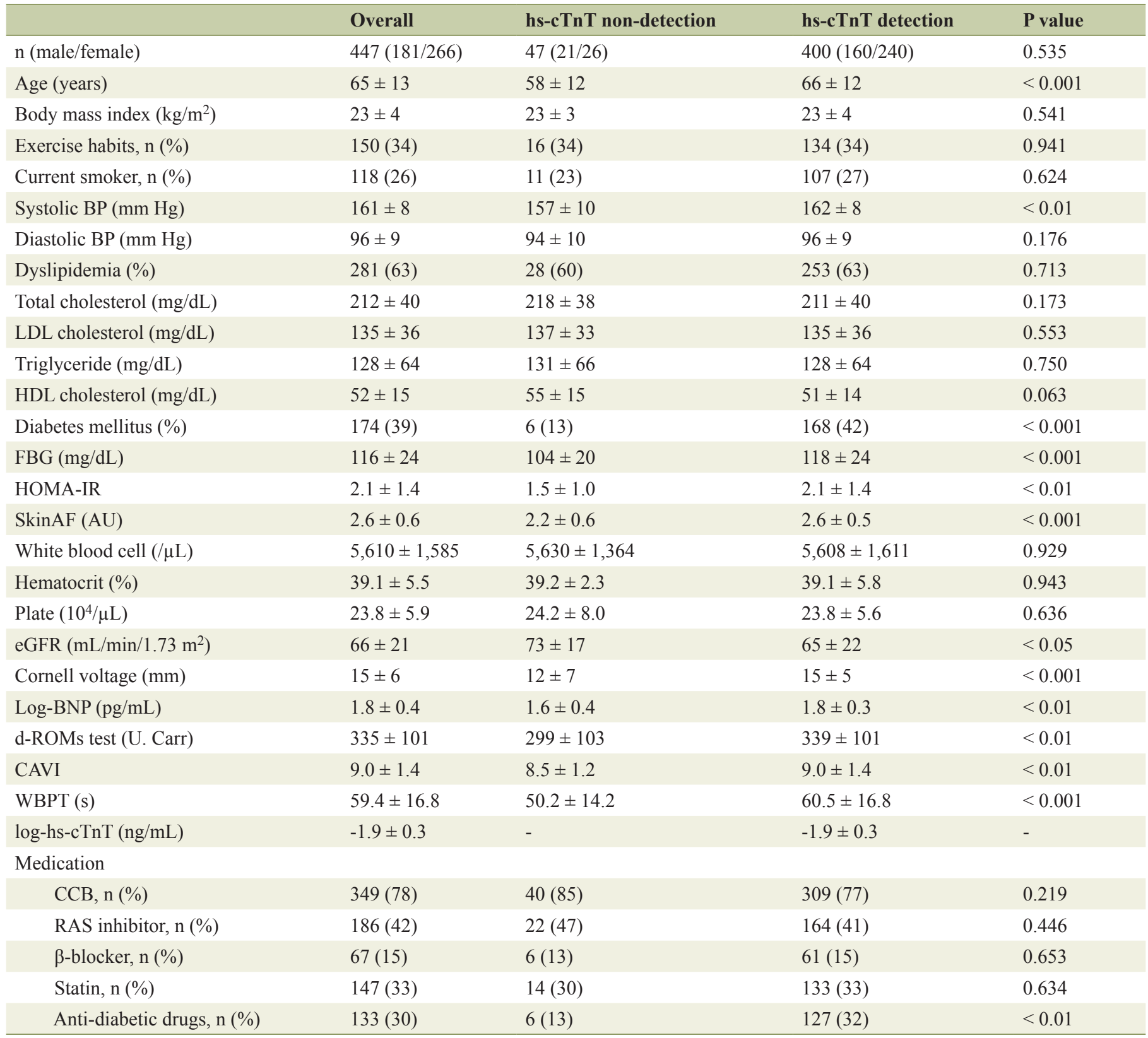

Data are expressed as mean \pm SD. hs-cTnT: high-sensitivity cardiac troponin T; BP: blood pressure; LDL: low-density lipoprotein; HDL: high-density lipoprotein; FBG: fasting blood glucose; HOMA-IR: homeostatic model assessment of insulin resistance; AF: autofluorescence; eGFR: estimated glomerular filtration rate; BNP: brain natriuretic peptide; d-ROMs: derivatives of reactive oxygen metabolites; CAVI: cardio-ankle vascular index; WBPT: whole blood passage time; CCB: calcium channel blocker; RAS: renin-angiotensin system.

gression of subclinical myocardial injury in patients with hypertension. Skin AF, CAVI and d-ROMs test were selected as independent variables for both hs-cTnT and WBPT as subordinate factors.

Hemorheology assessed by MC-FAN is an in vitro measurement that uses artificial blood vessels, with a 7- $\mu$ m wide, 30- $\mu \mathrm{m}$ long and 4.5- $\mu \mathrm{m}$ deep vessel lumen. Thus, assessment of hemorheology by MC-FAN is assumed to correspond to values obtained for small vessels. Small vessels are consid- ered to be present in a portion of skin biopsies along with systemic vessels, including heart vessels. On the contrary, the results of this study indicated that skin AF as a marker of AGEs in tissues was selected as an independent variable when hs-cTnT was a subordinate factor. In addition, skin AF had an independent association with WBPT. Skin AF is reported to reflect pentosidine levels, which are major components of AGEs on the volar side of the lower arm according to the results of skin biopsy [22]. Therefore, impairment of hemor- 
Table 2. Correlation Between hs-cTnT, WBPT and Clinical Parameters in hs-cTnT Detectable Patients

\begin{tabular}{|c|c|c|}
\hline & $\log -\mathrm{hs}-\mathrm{cTn} T / \mathrm{r}$ & WBPT/r \\
\hline Sex $($ female $=0$, male $=1)$ & $-0.11 *$ & 0.05 \\
\hline Age & $0.27 * * *$ & 0.08 \\
\hline Body mass index & 0.02 & $0.11 *$ \\
\hline Exercise habits $(\mathrm{No}=0$, Yes $=1)$ & 0.08 & $-0.16^{* *}$ \\
\hline Current smoker $(\mathrm{No}=0, \mathrm{Yes}=1)$ & 0.10 & $0.17 * *$ \\
\hline Systolic BP & 0.09 & 0.06 \\
\hline Diastolic BP & 0.07 & 0.03 \\
\hline Dyslipidemia $(\mathrm{No}=0, \mathrm{Yes}=1)$ & 0.02 & 0.01 \\
\hline Total cholesterol & 0.09 & 0.03 \\
\hline LDL cholesterol & 0.06 & 0.04 \\
\hline Triglyceride & 0.09 & 0.07 \\
\hline HDL cholesterol & -0.03 & -0.02 \\
\hline Diabetes mellitus $(\mathrm{No}=0, \mathrm{Yes}=1)$ & $0.16^{* *}$ & $0.11 *$ \\
\hline FBG & $0.11 *$ & 0.10 \\
\hline HOMA-IR & 0.09 & 0.09 \\
\hline Skin AF & $0.41 * * *$ & $0.21 * * *$ \\
\hline White blood cell & 0.08 & $0.11^{*}$ \\
\hline Hematocrit & 0.09 & $0.18 * * *$ \\
\hline Plate & 0.08 & 0.07 \\
\hline eGFR & $-0.19 * * *$ & $-0.14 * *$ \\
\hline Cornell voltage & $0.15^{* *}$ & 0.09 \\
\hline $\log -\mathrm{BNP}$ & $0.26^{* * *}$ & 0.08 \\
\hline d-ROMs test & $0.29 * * *$ & $0.29^{* * *}$ \\
\hline CAVI & $0.39 * * *$ & $0.37 * * *$ \\
\hline $\mathrm{CCB}(\mathrm{No}=0, \mathrm{Yes}=1)$ & -0.05 & -0.04 \\
\hline RAS inhibitor $(\mathrm{No}=0, \mathrm{Yes}=1)$ & -0.09 & -0.08 \\
\hline$\beta$-blocker $($ No $=0$, Yes $=1)$ & -0.04 & 0.03 \\
\hline Statin $($ No $=0$, Yes $=1)$ & -0.07 & -0.09 \\
\hline Anti-diabetic drugs $(\mathrm{No}=0, \mathrm{Yes}=1)$ & -0.09 & -0.06 \\
\hline
\end{tabular}

r: expressed correlation coefficient. ${ }^{*} P<0.05$, ${ }^{* *} P<0.01$, ${ }^{* *} P<0.001$. hs-cTnT: high-sensitivity cardiac troponin T; WBPT: whole blood passage time; BP: blood pressure; LDL: low-density lipoprotein; HDL: highdensity lipoprotein; FBG: fasting blood glucose; HOMA-IR: homeostatic model assessment of insulin resistance; AF: autofluorescence; eGFR: estimated glomerular filtration rate; BNP: brain natriuretic peptide; $d$ ROMs: derivatives of reactive oxygen metabolites; CAVI: cardio-ankle vascular index; CCB: calcium channel blocker; RAS: renin-angiotensin system.

heology is a possible cause of the accumulation of AGEs in systemic tissues, including the heart. In addition, Hofmann et al have clarified a significant relationship between AGE-modified cardiac tissue collagen and skin AF [23]. Several pathways by which AGEs or their receptors influence myocardial injury have been reported [24-26]. Furthermore, basic studies have indicated that AGEs influence hemorheology by mechanisms such as platelet aggregation, leukocyte-endothelial in-

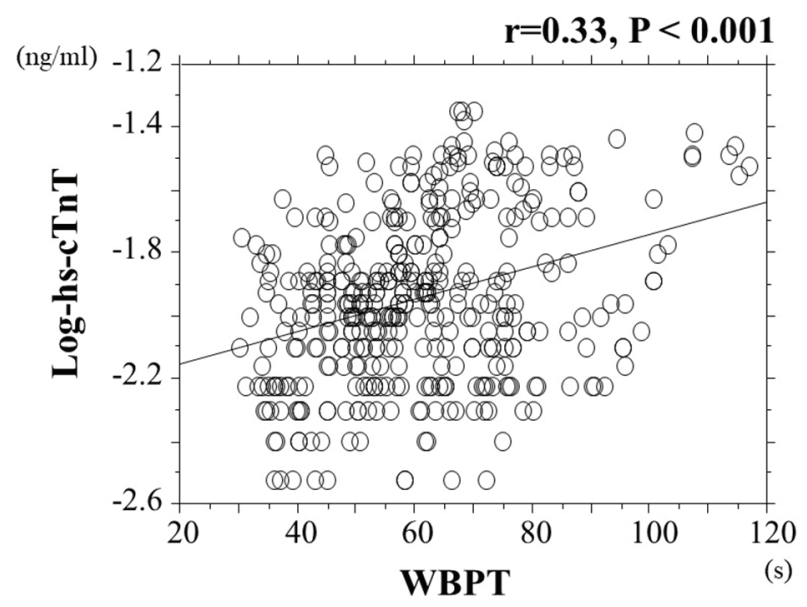

Figure 2. Relationship between hs-cTnT and WBPT. There is significantly positive correlation between log-hs-cTnT and WBPT ( $r=0.33$, $\mathrm{P}<0.001$ ). hs-cTnT: high-sensitivity troponin T; WBPT: whole blood passage time.

teraction and morphological changes in the erythrocyte membrane [27-29]. Thus, the results of this and previous studies indicate that hemorheology and AGEs are associated not only on the volar side of the lower arm but also in small cardiac vessels, consequently leading to myocardial injury in patients with hypertension.

CAVI is explored as a marker of arterial stiffness, which

Table 3. Multiple Regression Analysis

\begin{tabular}{lll} 
Explanatory factor & $\boldsymbol{\beta}$ & P value \\
\hline (A) Skin AF & 0.27 & $<0.001$ \\
CAVI & 0.16 & $<0.01$ \\
WBPT & 0.15 & $<0.01$ \\
Age & 0.13 & $<0.01$ \\
d-ROMs test & 0.12 & $<0.01$ \\
eGFR & -0.11 & $<0.01$ \\
log-BNP & 0.10 & $<0.05$ \\
Cornell voltage & 0.09 & 0.068 \\
(B) CAVI & 0.26 & $<0.001$ \\
log-hs-cTnT & 0.18 & $<0.01$ \\
d-ROMs test & 0.17 & $<0.01$ \\
Skin AF & 0.15 & $<0.01$ \\
Hematocrit & 0.13 & $<0.01$ \\
Exercise habits & -0.12 & $<0.01$ \\
Current smoker & 0.11 & $<0.05$ \\
eGFR & -0.09 & 0.057 \\
\hline
\end{tabular}

(A) Subordinate factor is log-hs-cTnT, $\mathrm{R}^{2}=0.35$; (B) subordinate factor is WBPT, $\mathrm{R}^{2}=0.30$. AF: autofluorescence; CAVI: cardio-ankle vascular index; WBPT: whole blood passage time; d-ROMs: derivatives of reactive oxygen metabolites; eGFR: estimated glomerular filtration rate; BNP: brain natriuretic peptide; hs-cTnT: high-sensitivity cardiac troponin $\mathrm{T}$. 


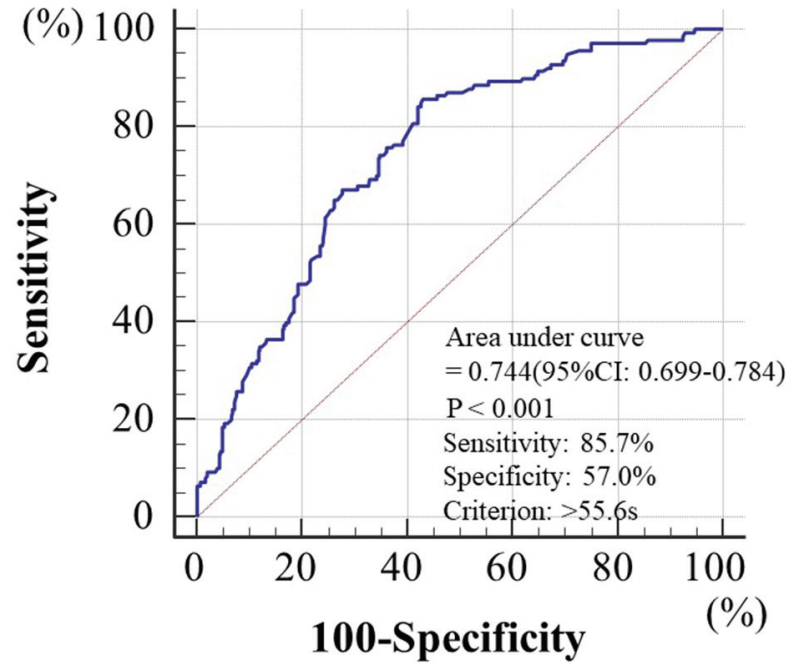

Figure 3. Cutoff value of WBPT for discriminating hs-cTnT levels as $\geq$ $0.014 \mathrm{ng} / \mathrm{mL}$. Receiver-operating characteristic indicated that a WBPT cutoff of $55.6 \mathrm{~s}$ yielded the largest area under the curve, $0.744(95 \%$ confidence interval: $0.699-0.784 ; P<0.001)$, indicating a sensitivity of $85.7 \%$ and a specificity of $57.0 \%$ for discriminating hs-cTnT levels as $\geq 0.014 \mathrm{ng} / \mathrm{mL}$. WBPT: whole blood passage time; hs-cTnT: highsensitivity troponin $\mathrm{T}$.

is independently associated with blood pressure [15]. Several recent studies have reported the significance of left ventricular dysfunction in the progression of myocardial injury. Furthermore, increases in aortic artery stiffness or arterial reflection wave are known to be caused by left ventricular dysfunction $[30,31]$. Therefore, the independent relationship between CAVI and hs-cTnT identified in this study is thought to reflect subclinical myocardial injury via left ventricular dysfunction resulting from an increase in afterload or arterial reflection wave. In addition, some researchers have reported that CAVI reflects endothelial function [32, 33]. Endothelial dysfunction has also been known to cause left ventricular dysfunction and myocardial injury $[34,35]$. Thus, the results of this study indicate the importance of arterial dysfunction in subclinical myocardial injury in patients with hypertension. CAVI was also selected as an independent variable for WBPT as a subordinate factor in multivariate analysis. Some studies have reported relationships between WBPT and arterial function, such as arterial stiffness, endothelial function and vascular resistance [9, $21,36,37]$. Therefore, increased WBPT due to increased hscTnT levels may be partly explained by arterial dysfunction.

A number of studies indicate that oxidative stress has a crucial role in the pathogenesis of hypertension $[38,39]$. The results of the present study also indicate that the d-ROMs test, as a marker of oxidative stress, is associated with both myocardial injury and hemorheology in patients with hypertension. Several pathways have been identified by which oxidative stress causes myocardial injury, such as dysfunction of the mitochondrial electron transport complex, activity of nicotinamide adenine dinucleotide phosphate oxidase, apoptosis of myocardial cells and nitric oxide synthase uncoupling [40-42]. In addition, several mechanisms are also reported by which oxidative stress causes impairment of hemorheology, such as platelet aggregation and elevation of plasma viscosity [43, 44]. Thus, oxidative stress is an important target factor for the prevention of both the progression of myocardial injury and impairment of hemorheology in patients with hypertension.

It is useful to have target cutoff levels of WBPT for predicting abnormal hs-cTnT levels in clinical settings. This study clarifies the clinical usefulness of assessing WBPT to detect hs-cTnT levels as high as $\geq 0.014 \mathrm{ng} / \mathrm{mL}$, which is shown to be discriminatory of increased hs-cTnT levels [45]. ROC curve analysis for hs-cTnT levels as high as $\geq 0.014 \mathrm{ng} / \mathrm{mL}$ indicated that a cutoff value for WBPT of $55.6 \mathrm{~s}$ yielded the largest area under the curve (0.744) for discriminating high hs-cTnT levels. Although this was a cross-sectional study, we believe that it is possible to evaluate the risk of the progression of myocardial injury in patients with hypertension by measuring WBPT. Some clinical studies have indicated that medication, exercise habits and smoking cessation improve hemorheology [46-51]. In this cross-sectional study, exercise habits and current smoking status were independently associated with WBPT, although no significant association was observed between medication and WBPT. We suggest that progression of myocardial injury can be prevented by interventions, such as lifestyle modification or aggressive use of medication, which is effective to improve hemorheology in hypertensive patients to maintain WBPT at approximately $\leq 55 \mathrm{~s}$.

This study has several limitations. First, the contents of medical treatment may have influenced the study results. Second, angiography, computed tomography, magnetic resonance imaging and echocardiography were not performed; thus, asymptomatic cardiovascular diseases may have remained undetected. Third, assessment of hemorheology by MC-FAN is an in vitro method that uses artificial blood vessels; therefore, the hemorheological data obtained might differ from those obtained in vivo because of the influence of vascular factors, such as endothelial or smooth muscle cells. However, the results of this study indicate that WBPT is a considerable risk factor for the progression of subclinical myocardial injury in patients with hypertension. Finally, this study was cross-sectional and conducted in a single unit with a relatively small sample size. Future multicenter prospective studies, including intervention therapies, will be required to confirm the results of this study.

\section{Conclusions}

WBPT is independently associated with hs-cTnT in hypertensive patients with no history of cardiovascular events, suggesting that impairment of hemorheology in small cardiac vessels causes subclinical myocardial injury. In addition, this study suggests that the progression of myocardial injury can be prevented by maintaining WBPT at approximately $\leq 55 \mathrm{~s}$. Additional prospective studies, including intervention therapies, will be required to confirm the results of this study.

\section{Conflict of Interest}

The author has no competing interests. 


\section{Grant Support}

None.

\section{Financial Disclosure}

The author has reported no conflicts of interest.

\section{References}

1. Twerenbold R, Jaffe A, Reichlin T, Reiter M, Mueller C. High-sensitive troponin T measurements: what do we gain and what are the challenges? Eur Heart J. 2012;33(5):579-586.

2. Sato Y, Yamamoto E, Sawa T, Toda K, Hara T, Iwasaki T, Fujiwara $\mathrm{H}$, et al. High-sensitivity cardiac troponin $\mathrm{T}$ in essential hypertension. J Cardiol. 2011;58(3):226-231.

3. Miao DM, Zhang LP, Yu HP, Zhang JY, Xiao WK, Ye P. Serum levels of high-sensitivity troponin T: a novel marker for left ventricular remodeling and performance in hypertensive subjects. Genet Mol Res. 2014;13(3):51435153.

4. Cayli M, Gur M, Elbasan Z, Seker T, Turkoglu C, Kaypakli O, Sahin DY, et al. High-sensitivity cardiac troponin $\mathrm{T}$ predicts nondipper hypertension in newly diagnosed hypertensive patients. J Clin Hypertens (Greenwich). 2013;15(10):731-736.

5. Hitsumoto T. Arterial Velocity Pulse Index as a Novel Marker of Atherosclerosis Using Pulse Wave Analysis on High Sensitivity Troponin T in Hypertensive Patients. Cardiol Res. 2017;8(2):36-43.

6. Tzoulaki I, Murray GD, Lee AJ, Rumley A, Lowe GD, Fowkes FG. Relative value of inflammatory, hemostatic, and rheological factors for incident myocardial infarction and stroke: the Edinburgh Artery Study. Circulation. 2007;115(16):2119-2127.

7. Cowan AQ, Cho DJ, Rosenson RS. Importance of blood rheology in the pathophysiology of atherothrombosis. Cardiovasc Drugs Ther. 2012;26(4):339-348.

8. Kikuchi Y, Sato K, Mizuguchi Y. Modified cell-flow microchannels in a single-crystal silicon substrate and flow behavior of blood cells. Microvasc Res. 1994;47(1):126139.

9. Satoh N, Kotani K, Wada H, Himeno A, Shimada S, Sasaki Y, Yamada K, et al. Unfavorable blood rheology is closely associated with arterial stiffness in obese patients. Endocr J. 2009;56(7):915-918.

10. Hitsumoto T. Factors affecting impairment of blood rheology in obese subjects. J Cardiol. 2012;60(5):401-406.

11. Yoshida K, Kimura T, Aoki T, Tsunekawa K, Araki O, Shoho Y, Nara M, et al. Fasting serum insulin levels and insulin resistance are associated with blood rheology in Japanese young adults without diabetes. J Int Med Res. 2016;44(3):496-507.

12. Hitsumoto T. Usefulness of the Whole Blood Passage Time as a Predictor of Primary Cardiovascular Events in
Patients With Traditional Cardiovascular Risk Factors. Cardiol Res. 2018;9(4):231-238.

13. Meerwaldt R, Links TP, Graaff R, Hoogenberg $\mathrm{K}$, Lefrandt JD, Baynes JW, Gans RO, et al. Increased accumulation of skin advanced glycation end-products precedes and correlates with clinical manifestation of diabetic neuropathy. Diabetologia. 2005;48(8):1637-1644.

14. Okin PM, Devereux RB, Liu JE, Oikarinen L, Jern S, Kjeldsen SE, Julius S, et al. Regression of electrocardiographic left ventricular hypertrophy predicts regression of echocardiographic left ventricular mass: the LIFE study. J Hum Hypertens. 2004;18(6):403-409.

15. Shirai K, Utino J, Otsuka K, Takata M. A novel blood pressure-independent arterial wall stiffness parameter; cardio-ankle vascular index (CAVI). J Atheroscler Thromb. 2006;13(2):101-107.

16. Friedewald WT, Levy RI, Fredrickson DS. Estimation of the concentration of low-density lipoprotein cholesterol in plasma, without use of the preparative ultracentrifuge. Clin Chem. 1972;18(6):499-502.

17. Matthews DR, Hosker JP, Rudenski AS, Naylor BA, Treacher DF, Turner RC. Homeostasis model assessment: insulin resistance and beta-cell function from fasting plasma glucose and insulin concentrations in man. Diabetologia. 1985;28(7):412-419.

18. Imai E, Horio M, Nitta K, Yamagata K, Iseki K, Hara $\mathrm{S}$, Ura $\mathrm{N}$, et al. Estimation of glomerular filtration rate by the MDRD study equation modified for Japanese patients with chronic kidney disease. Clin Exp Nephrol. 2007;11(1):41-50.

19. Cesarone MR, Belcaro G, Carratelli M, Cornelli U, De Sanctis MT, Incandela L, Barsotti A, et al. A simple test to monitor oxidative stress. Int Angiol. 1999;18(2):127-130.

20. Mingels A, Jacobs L, Michielsen E, Swaanenburg J, Wodzig W, van Dieijen-Visser M. Reference population and marathon runner sera assessed by highly sensitive cardiac troponin $\mathrm{T}$ and commercial cardiac troponin $\mathrm{T}$ and I assays. Clin Chem. 2009;55(1):101-108.

21. Yagi H, Sumino H, Aoki T, Tsunekawa K, Araki O, Kimura T, Nara M, et al. Impaired blood rheology is associated with endothelial dysfunction in patients with coronary risk factors. Clin Hemorheol Microcirc. 2016;62(2):139150 .

22. Meerwaldt R, Graaff R, Oomen PHN, Links TP, Jager JJ, Alderson NL, Thorpe SR, et al. Simple non-invasive assessment of advanced glycation endproduct accumulation. Diabetologia. 2004;47(7):1324-1330.

23. Hofmann B, Jacobs K, Navarrete Santos A, Wienke A, Silber RE, Simm A. Relationship between cardiac tissue glycation and skin autofluorescence in patients with coronary artery disease. Diabetes Metab. 2015;41(5):410-415.

24. Ma H, Li SY, Xu P, Babcock SA, Dolence EK, Brownlee $\mathrm{M}, \mathrm{Li}$ J, et al. Advanced glycation endproduct (AGE) accumulation and AGE receptor (RAGE) up-regulation contribute to the onset of diabetic cardiomyopathy. J Cell Mol Med. 2009;13(8B):1751-1764.

25. Brett J, Schmidt AM, Yan SD, Zou YS, Weidman E, Pinsky D, Nowygrod R, et al. Survey of the distribution of a newly characterized receptor for advanced glycation 
end products in tissues. Am J Pathol. 1993;143(6):16991712 .

26. Yu Y, Wang L, Delguste F, Durand A, Guilbaud A, Rousselin C, Schmidt AM, et al. Advanced glycation end products receptor RAGE controls myocardial dysfunction and oxidative stress in high-fat fed mice by sustaining mitochondrial dynamics and autophagy-lysosome pathway. Free Radic Biol Med. 2017;112:397-410.

27. Hasegawa Y, Suehiro A, Higasa S, Namba M, Kakishita E. Enhancing effect of advanced glycation end products on serotonin-induced platelet aggregation in patients with diabetes mellitus. Thromb Res. 2002;107(6):319-323.

28. Morigi M, Angioletti S, Imberti B, Donadelli R, Micheletti G, Figliuzzi M, Remuzzi A, et al. Leukocyte-endothelial interaction is augmented by high glucose concentrations and hyperglycemia in a NF-kB-dependent fashion. J Clin Invest. 1998;101(9):1905-1915.

29. Awasthi S, Gayathiri SK, Ramya R, Duraichelvan R, Dhason A, Saraswathi NT. Advanced Glycation-Modified Human Serum Albumin Evokes Alterations in Membrane and Eryptosis in Erythrocytes. Appl Biochem Biotechnol. 2015;177(5):1013-1024.

30. Jaroch J, Loboz Grudzien K, Bociaga Z, Kowalska A, Kruszynska E, Wilczynska M, Dudek K. The relationship of carotid arterial stiffness to left ventricular diastolic dysfunction in untreated hypertension. Kardiol Pol. 2012;70(3):223-231.

31. Higashi H, Okayama H, Saito M, Morioka H, Aono J, Yoshii T, Hiasa G, et al. Relationship between augmentation index and left ventricular diastolic function in healthy women and men. Am J Hypertens. 2013;26(11):12801286.

32. Endo K, Saiki A, Ohira M, Miyashita Y, Shirai K. Cardioankle vascular index may reflect endothelial function in type 2 diabetes. Int J Clin Pract. 2011;65(11):1200-1201.

33. Kim KJ, Lee BW, Kim HM, Shin JY, Kang ES, Cha BS, Lee EJ, et al. Associations between cardio-ankle vascular index and microvascular complications in type 2 diabetes mellitus patients. J Atheroscler Thromb. 2011;18(4):328336.

34. Zizek B, Poredos P. Increased left ventricular mass and diastolic dysfunction are associated with endothelial dysfunction in normotensive offspring of subjects with essential hypertension. Blood Press. 2007;16(1):36-44.

35. Marti V, Aymat R, Ballester M, Garcia J, Carrio I, Auge JM. Coronary endothelial dysfunction and myocardial cell damage in chronic stable idiopathic dilated cardiomyopathy. Int J Cardiol. 2002;82(3):237-245.

36. Hitsumoto T. Impact of hemorheology assessed by the microchannel method on pulsatility index of the common carotid artery in patients with type 2 diabetes mellitus. J Clin Med Res. 2017;9(7):579-585.

37. Hitsumoto T. Relationship between hemorheology assessed using microchannel array flow analyzer and kidney function in hypertensive patients. Cardiol Res. 2017;8(4):147-153.

38. Sinha N, Dabla PK. Oxidative stress and antioxidants in hypertension-a current review. Curr Hypertens Rev. 2015;11(2):132-142.

39. Montezano AC, Dulak-Lis M, Tsiropoulou S, Harvey A, Briones AM, Touyz RM. Oxidative stress and human hypertension: vascular mechanisms, biomarkers, and novel therapies. Can J Cardiol. 2015;31(5):631-641.

40. Li SY, Yang X, Ceylan-Isik AF, Du M, Sreejayan N, Ren $\mathrm{J}$. Cardiac contractile dysfunction in Lep/Lep obesity is accompanied by NADPH oxidase activation, oxidative modification of sarco(endo)plasmic reticulum Ca2+-ATPase and myosin heavy chain isozyme switch. Diabetologia. 2006;49(6):1434-1446.

41. Boudina S, Abel ED. Diabetic cardiomyopathy, causes and effects. Rev Endocr Metab Disord. 2010;11(1):31-39.

42. Ansley DM, Wang B. Oxidative stress and myocardial injury in the diabetic heart. J Pathol. 2013;229(2):232-241.

43. Tzoulaki I, Murray GD, Lee AJ, Rumley A, Lowe GD, Fowkes FG. Inflammatory, haemostatic, and rheological markers for incident peripheral arterial disease: Edinburgh Artery Study. Eur Heart J. 2007;28(3):354-362.

44. Podrez EA, Byzova TV, Febbraio M, Salomon RG, Ma Y, Valiyaveettil M, Poliakov E, et al. Platelet CD36 links hyperlipidemia, oxidant stress and a prothrombotic phenotype. Nat Med. 2007;13(9):1086-1095.

45. Saunders JT, Nambi V, de Lemos JA, Chambless LE, Virani SS, Boerwinkle E, Hoogeveen RC, et al. Cardiac troponin $\mathrm{T}$ measured by a highly sensitive assay predicts coronary heart disease, heart failure, and mortality in the Atherosclerosis Risk in Communities Study. Circulation. 2011;123(13):1367-1376

46. Yamada K, Hirayama T, Hasegawa Y. Antiplatelet effect of losartan and telmisartan in patients with ischemic stroke. J Stroke Cerebrovasc Dis. 2007;16(5):225-231.

47. Shamanaev AY, Aliev OI, Anishchenko AM, Sidehmenova AV, Plotnikov MB. Hemorheological effects of amlodipine in spontaneously hypertensive rats. Indian J Pharmacol. 2017;49(4):312-316.

48. Ott C, Raff U, Schmidt S, Kistner I, Friedrich S, Bramlage P, Harazny JM, et al. Effects of saxagliptin on early microvascular changes in patients with type 2 diabetes. Cardiovasc Diabetol. 2014;13:19.

49. Tirnaksiz E, Pamukcu B, Oflaz H, Nisanci Y. Effect of high dose statin therapy on platelet function; statins reduce aspirin-resistant platelet aggregation in patients with coronary heart disease. J Thromb Thrombolysis. 2009;27(1):24-28.

50. Coppola L, Grassia A, Coppola A, Tondi G, Peluso G, Mordente S, Gombos G. Effects of a moderate-intensity aerobic program on blood viscosity, platelet aggregation and fibrinolytic balance in young and middle-aged sedentary subjects. Blood Coagul Fibrinolysis. 2004;15(1):3137.

51. Shimada S, Hasegawa K, Wada H, Terashima S, SatohAsahara N, Yamakage H, Kitaoka S, et al. High blood viscosity is closely associated with cigarette smoking and markedly reduced by smoking cessation. Circ J. 2011;75(1):185-189. 